\title{
Cytogenetic Analysis of a Pseudoangiomatous Pleomorphic/Spindle Cell Lipoma
}

\author{
IOANNIS PANAGOPOULOS ${ }^{1}$, LUDMILA GORUNOVA ${ }^{1}$, INGVILD LOBMAIER ${ }^{2}$, \\ HEGE KILEN ANDERSEN $^{1}$, BODIL BJERKEHAGEN ${ }^{2}$ and SVERRE HEIM ${ }^{1,3}$ \\ ${ }^{1}$ Section for Cancer Cytogenetics, Institute for Cancer Genetics and Informatics, \\ The Norwegian Radium Hospital, Oslo University Hospital, Oslo, Norway; \\ ${ }^{2}$ Department of Pathology, The Norwegian Radium Hospital, Oslo University Hospital, Oslo, Norway; \\ ${ }^{3}$ Faculty of Medicine, University of Oslo, Oslo, Norway
}

\begin{abstract}
Background: Pseudoangiomatous pleomorphic/ spindle cell lipoma is a rare subtype of pleomorphic/spindle cell lipoma. Only approximately 20 such tumors have been described. Genetic information on pseudoangiomatous pleomorphic/spindle cell lipoma is restricted to a single case in which deletion of the forkhead box O1 (FOXO1) gene was found, using fluorescence in situ hybridization (FISH). Materials and Methods: G-banding and FISH analyses were performed on a pseudoangiomatous pleomorphic/spindle cell lipoma. Results: G-banding of tumor cells showed complex karyotypic changes including loss of chromosome 13. FISH analysis revealed that the deleted region contained the $R B 1$ gene (13q14.2) and the part of chromosome arm 13q (q14.2q14.3) in which spans the TRIM13 gene, the two non-coding RNA genes, DLEU1 and DLEU2, and the genetic markers RH44686 and D13S25. Conclusion: Several acquired genomic aberrations were found in the tumor. Among them was loss of chromosome 13 material. Results confirm the (cyto)genetic similarity between pseudoangiomatous pleomorphic/spindle cell lipoma and spindle cell lipomas.
\end{abstract}

Pseudoangiomatous pleomorphic/spindle cell lipoma is a rare subtype of pleomorphic/spindle cell lipoma first described by Hawley et al. in 1994 in five patients (1). The tumors had 'the features of typical spindle cell lipoma but, in addition, exhibited irregular and branching spaces with villiform connective tissue projections, giving a striking angiomatoid

Correspondence to: Ioannis Panagopoulos, Section for Cancer Cytogenetics, Institute for Cancer Genetics and Informatics, The Norwegian Radium Hospital, Oslo University Hospital, P.O.Box 4953 Nydalen, NO-0424 Oslo, Norway. Tel: +47 22782363, e-mail: ioannis.panagopoulos@rr-research.no

Key Words: Pseudoangiomatous pleomorphic/spindle cell lipoma, cytogenetics, chromosome 13, deletion, 13q14, RB1, FOXO1. appearance' (1). To date, only 20 patients have been described in the literature with this diagnosis, 15 of whom were males (1-10). The pseudoangiomatous pleomorphic/spindle cell lipomas were mostly found in the neck (seven patients) and shoulders (four patients), but have also been seen in the cheek, chest, chin, elbow, finger, subscapular region, and thumb. Genetic information on pseudoangiomatous pleomorphic/ spindle cell lipoma is restricted to one case only (8) in which fluorescence in situ hybridization (FISH) with a probe for the forkhead box O1 (FOXO1) gene, which maps to chromosome sub-band $13 \mathrm{q} 14.11$, showed a signal pattern indicating monoallelic loss of the gene in $57 \%$ of the examined cells.

We present here cytogenetic data on another case of pseudoangiomatous pleomorphic/spindle cell lipoma which showed loss of 13q material.

\section{Case Report}

Ethics statement. The study was approved by the Regional Committee for Medical and Health Research Ethics, SouthEast Norway (Regional komité for medisinsk forskningsetikk Sor-Ost, Norge, http://helseforskning .etikkom.no, REC no S07474a). All patient information has been de-identified.

Patient. A 35-year-old man noticed a tumor of his left elbow after a trauma. It seemed to grow over 2 years, when a magnetic resonance imaging investigation measured it at $6 \mathrm{~cm}$ in diameter. The subcutaneous tumor contained fatty and myxoid tissues and was surgically removed by marginal excision.

Macroscopically, the encapsulated tumor's yellow cut surface was partly myxoid, partly fleshy. Microscopically, the appearance was mixed, with mature fat cells intermingled with bands of fibrous, partly myxoid tissue, bundles of 'ropey' collagen and small, spindled cells that mostly lacked atypia (Figure 1A-C). In some areas, however, atypically-appearing cells and scattered floret-like 

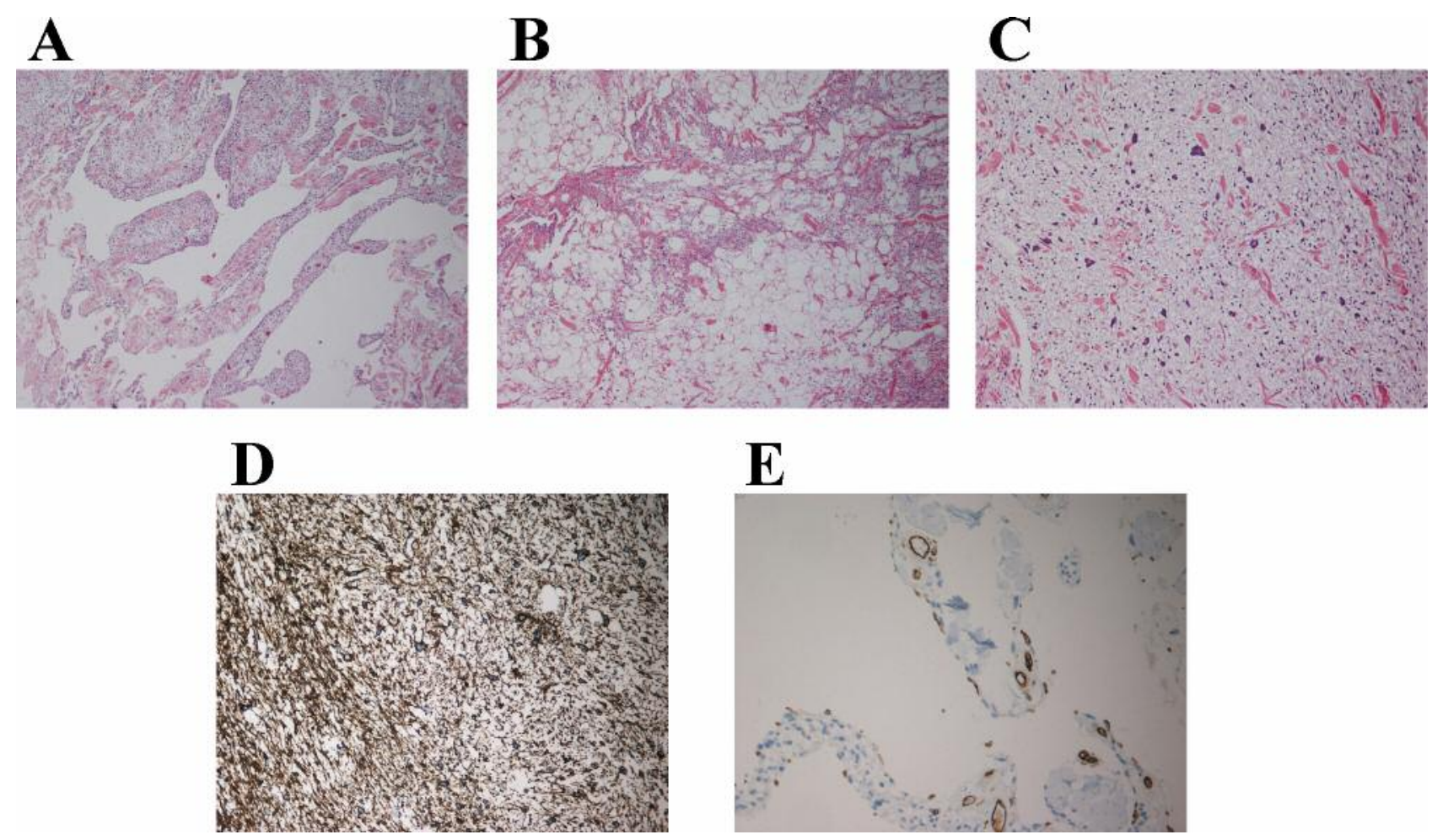

Figure 1. Histological examination of the pseudoangiomatous pleomorphic/spindle cell lipoma. A: Pseudoangiomatous cavity [ $\times 4$ hematoxylin and eosin stain $(H E)$ ]. B: Lipoma transition to spindle cell lipoma $(\times 4$ HE). C: Pleomorphic/spindle cell lipoma with floret cells $(\times 10$ HE). D: Immunohistochemical analysis showing positivity for CD34 (×10). E: Immunohistochemical analysis demonstrating focal CD31-positive cells along the cavities ( $\times 20)$.

giant cells were seen (Figure 1B-C). Centrally, the tumor had a pseudoangiomatous appearance, with papillary formations surrounding the vascular spaces (Figure 1A). There was no necrosis and the mitotic activity was low (Ki67 index <5\%).

Immunohistochemical analysis showed extensive positivity for CD34 in the fibrous tissue (Figure 1D). The fat cells were S100-positive (Figure 1E). The angiomatous areas were partly CD31-positive, but negative for podoplanin (using D2-40). MDM2 proto-oncogene (MDM2) staining was negative.

The combination of 'ropey' collagen, fibrous tissue with no atypia, and floret-like giant cells fits very well with a spindle cell or pleomorphic lipoma. The rare pseudoangiomatous growth led to the final diagnosis of pseudoangiomatous pleomorphic/spindle cell lipoma.

G-Banding and karyotyping. As part of our diagnostic service, fresh tissue from a representative area of the tumor was received and analyzed cytogenetically after short-term culturing as described elsewhere (11). The karyotype was described according to the International System for Human Cytogenetic Nomenclature 2016 guidelines (12).
FISH analysis. The following three probes were used for FISH analysis. I: Vysis LSI FOXO1 (13q14) Dual Color Break Apart Rearrangement Probe (Abbott Laboratories, Abbott Park, IL, USA). This probe hybridizes to a $1.5 \mathrm{Mbp}$ sequence on 13q14.11 which contains the FOXO1 gene. II: $\mathrm{RB}$ transcriptional corepressor $1(R B I)$ deletion probe, purchased from Cytocell (Cytocell, Cambridge, UK), was used to detect deletion of the $R B 1$ locus in $13 \mathrm{q} 14.2$. It consists of a $318-\mathrm{kb}$ red probe spanning $R B 1$ and a $13 \mathrm{qter}$ green probe acting as a control for chromosome 13. III: The $13 \mathrm{q} 14.3$ deletion probe from Cytocell. This probe is normally used for detection of 13q14 deletion in B-cell chronic lymphocytic leukemia. It spans the tripartite motif containing 13 (TRIM13) gene, two non-coding RNA genes, deleted in lymphocytic leukemia 1 (DLEUI) and deleted in lymphocytic leukemia 2 (DLEU2), and the genetic marker D13S319. It also contains a 13qter subtelomere specific probe (clone 163C9), labeled in green, which allows for identification of chromosome 13 and acts as a control.

Fluorescent signals were captured and analyzed using the CytoVision system from Leica Biosystems (http:// www.leicabiosystems.com/pathology-imaging/cytogenetics/). 


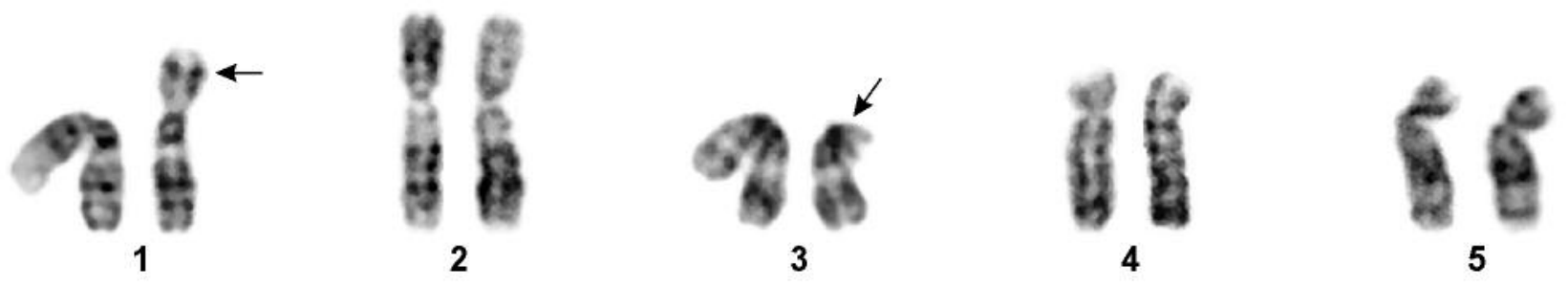

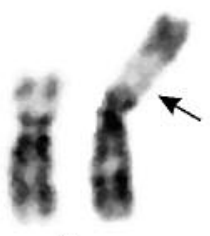

6

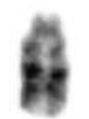

13

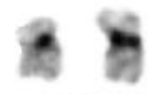

19

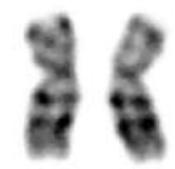

7

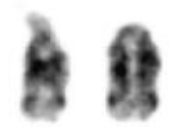

14

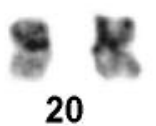

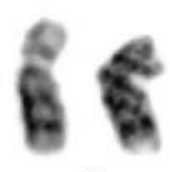

8

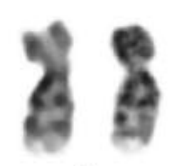

9

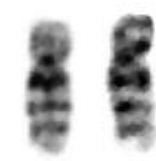

10

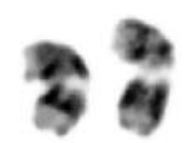

11

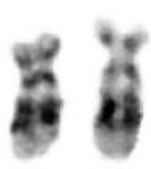

12

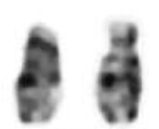

15

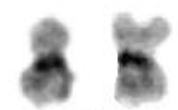

16

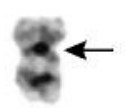

17

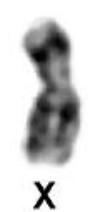

18
21
22

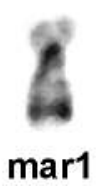

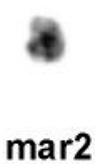

mar2

Figure 2. G-Banding analysis of the pseudoangiomatous pleomorphic/spindle cell lipoma. Karyotype showing the chromosomal aberrations of tumor cells: 45,XY,del(1)(p32),add(3)(p13),ins(6;?)(p21;?),-13,der(17;22)(q10;q10),-17,-22,+der(?)t(3;?)(p13;?),+mar1,+mar2 [14]. Arrows indicate breakpoints.

Findings. G-Banding analysis of short-term cultured cells from the pseudoangiomatous pleomorphic/spindle cell lipoma yielded the karyotype 45,XY,del(1)(p32), add(3)(p13),ins(6;?) (p21;?),-13,der(17;22)(q10;q10),-17,-22,+der(?)t $(3 ; ?)$ (p13;?),+mar1,+mar2[14] (Figure 2).

Interphase FISH was performed, using commercially available probes, for the $F O X O 1, R B 1$, and TRIM13 genes, the two DLEU1 and DLEU2 non-coding RNA genes, and the genetic markers RH44686 and D13S25 (Figure 3A). All of them are mapped on chromosome band 13q14 to different sub-bands (Figure 3A).

No loss of the FOXO1 probe (sub-band 13q14.11) was seen in the 115 nuclei examined (Figure 3B). However, FISH showed heterozygous loss of the RBI probe (sub-band 13q14.2). In 102 out of 112 (91\%) nuclei examined, one red signal (probe spanning $R B 1$ ) and two green signals (13qter probe) were seen (Figure 3C). Similarly, heterozygous loss of the region spanning TRIM13, DLEU1, DLEU2, and the genetic markers RH44686 and D13S25 was found (sub-bands 13q14.2-q14.3). In 92 out of 98 (94\%) interphase nuclei, one red signal (probe spans TRIM13, DLEU1, DLEU2, RH44686, and D13S25) and two green (13qter subtelomere specific probe) signals were observed (Figure 3D).

\section{Discussion}

The present study described the first karyotypic analysis of a pseudoangiomatous pleomorphic/spindle cell lipoma. Several clonal aberrations were found, including what at first seemed to be loss of one copy of chromosome 13, and FISH confirmed the heterozygous loss of $13 \mathrm{q} 14$ markers. This is in agreement with the cytogenetic pattern seen in most of the 28 karyotypically abnormal spindle cell lipomas reported in the literature, i.e. loss of material from $13 \mathrm{q}$ together with 


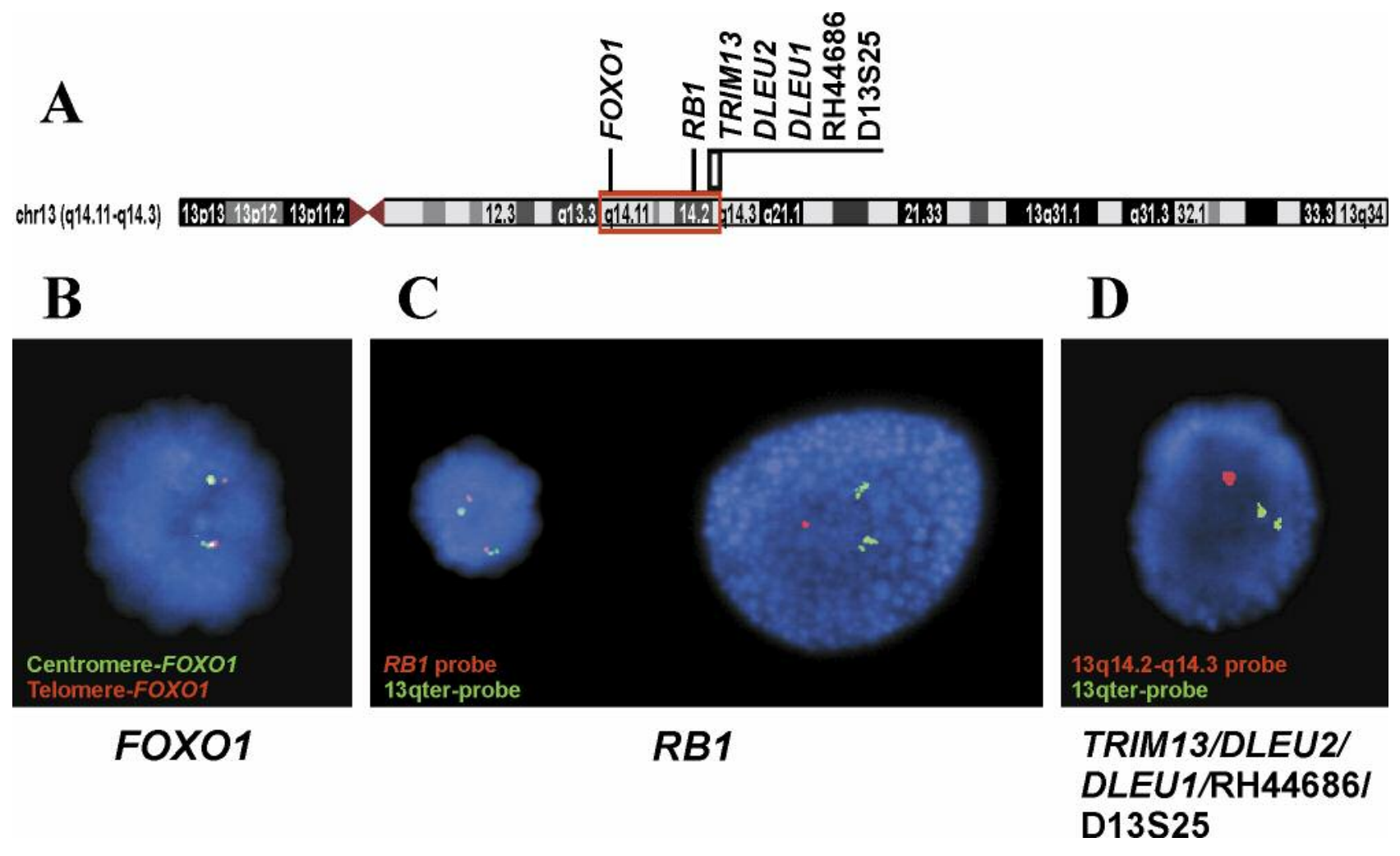

Figure 3. Interphase fluorescence in-situ hybridization (FISH) analysis of the pseudoangiomatous pleomorphic/spindle cell lipoma. A: Ideogram of chromosome 13 showing the mapping position of forkhead box O1 (FOXO1), RB transcriptional corepressor 1 (RB1), tripartite motif containing 13 (TRIM13), deleted in lymphocytic leukemia 2 (DLEU2), deleted in lymphocytic leukemia 1 (DLEU1), RH44686 and D13S25. B: FISH with the dual-color break-apart probe for FOXO1. The analysis showed no deletion of FOXO1. C: FISH with the RB1 deletion probe which consisted of a red probe spanning RB1 and a 13qter green probe. Left: A nucleus with normal (two red and two green) signals for RB1. Right: A nucleus with heterozygous deletion of RB1 (one red and two green signals). D: FISH with the 13q14.3 deletion probe. It consisted of a red probe (13q14.2-q14.3) spanning TRIM13, DLEU1, DLEU2, and the genetic markers RH44686 and D13S2, and a 13qter subtelomeric green probe. The analysis showed heterozygous deletion of the TRIM13/DLEU2/DLEU1/RH44686/D13S25 (red signal) region, whereas the region of chromosome 13 which was hybridized to 13qter green probe, was not deleted.

other chromosome anomalies (13-19). In a previous study, Forcucci et al. used a FOXO1 break-apart probe to find monoalleleic loss of the FOXO1 locus in $57 \%$ of the studied cells (8). In contrast, we found no loss of FOXOl using the same probe. On the other hand, our analysis showed heterozygous loss of the $R B 1$ locus and of a 13q14.2-q14.3 region in which spans the TRIM13 gene, two non-coding RNA genes, DLEU1 and DLEU2, and the genetic markers RH44686 and D13S25. However, 13qter was not found to be deleted, as shown by retention of the control green probe, indicating that chromosome 13 was not lost in its entirety. We assume that $13 \mathrm{q}$ material not found to be lost by FISH must be hidden in the $\operatorname{der}(?) \mathrm{t}(3 ; ?)$ or the marker chromosomes that were also part of the karyotype.

FISH analyses with probes for the $F O X O 1$ and $R B 1$ genes were in the past performed to demonstrate loss of $13 \mathrm{q}$ in spindle cell lipomas and related tumors $(14,15,20,21)$.
Maggiani et al. examined two cellular angiofibromas by interphase FISH (20). The first tumor had a deletion of $13 \mathrm{q} 14$ that included the RBI and FOXO1 loci. In the second tumor, the deletion contained FOXOl but not RBI (20). The divergent findings between the present study and that of Forcucci et al. (8) with regard to loss of the $\mathrm{FOXO1}$ locus may reflect the difference between partial (present case) and complete heterozygous loss of chromosome 13 (8).

Studies of spindle cell lipomas have identified two minimal deleted regions (MDR) in 13q14 (14, 15). In MDR1 (13q14.2), the genes $R B 1$, lysophosphatidic acid receptor 6 (LPAR6), RCC1 and BTB domain containing protein 2 (RCBTB2), and cysteinyl leukotriene receptor 2 (CYSLTR2) are located (chr13:48,868,089-49,294,385). MDR2 lies between the genes fibronectin type III domain containing $3 \mathrm{~A}$ (FNDC3A) and transmembrane phosphoinositide 3-phosphatase and tensin homolog 2 pseudogene 3 (TPTE2P3, formerly LOC220115). It 
is approximately $3600-\mathrm{Mbp}$ long (chr13:49,550,048$53,269,926)$ and has more than 30 gene loci (among them $D L E U 1$ and DLEU2) as well as the two microRNA genes $m i R$ $15 a$ and $m i R-16-1$ (14). Based on the expression of $R B 1$, which was not significantly reduced, and the absence of mutations, the authors concluded that there was no decisive support for $R B 1$ as the main target for chromosomal deletions in spindle cell lipomas. Instead, their data implicated miR-16-1 as a potential target (14). On the other hand, immunohistochemical staining showed that nuclear RB1 expression was deficient in all examined spindle cell lipomas, pleomorphic lipomas, and cellular angiofibromas, as well as in 17 out of 19 (89\%) mammary-type myofibroblastomas (22). At the moment, the molecular target of chromosome 13 aberrations remains unknown in these as well as most other tumors.

In conclusion, our study showed several acquired chromosomal changes including heterozygous loss of $13 q$ material in pseudoangiomatous pleomorphic/spindle cell lipoma. The deleted region contained the $R B 1$ gene and the part of chromosome arm 13q (q14.2-q14.3) which spans the TRIM13 gene, the two non-coding RNA genes, DLEU1 and DLEU2, and the genetic markers RH44686 and D13S25.

\section{Conflicts of Interests}

The Authors declare that they have no competing interests.

\section{Acknowledgements}

This work was supported by grants from the Norwegian Radium Hospital Foundation.

\section{References}

1 Hawley IC, Krausz T, Evans DJ and Fletcher CD: Spindle cell lipoma-a pseudoangiomatous variant. Histopathology 24: 565569,1994

2 Richmond I and Banerjee SS: Spindle cell lipoma - a pseudoangiomatous variant. Histopathology 27: 201, 1995.

3 Zamecnik M: Pseudoangiomatous spindle cell lipoma with 'true' angiomatous features. Virchows Arch 447: 781-783, 2005.

4 Billings SD and Folpe AL: Diagnostically challenging spindle cell lipomas: a report of 34 'low-fat' and 'fat-free' variants. Am J Dermatopathol 29: 437-442, 2007.

5 Zamecnik M and Michal M: Angiomatous spindle cell lipoma: Report of three cases with immunohistochemical and ultrastructural study and reappraisal of former 'pseudoangiomatous' variant. Pathol Int 57: 26-31, 2007.

6 Zouaidia F and Coindre JM: Pseudoangiomatous spindle cell lipoma: about six cases. Ann Pathol 31: 242-245, 2011 (in French)

7 Dong BW, Ma XY, He HN and Zhang FJ: Pseudoangiomatous variant of spindle cell lipoma: report of a case. Zhonghua Bing Li Xue Za Zhi 42: 343-344, 2013 (in Chinese).

8 Forcucci JA, Sugianto JZ, Wolff DJ, Maize JC, Sr. and Ralston JS: 'Low-fat' pseudoangiomatous spindle cell lipoma: a rare variant with loss of 13q14 region. Am J Dermatopathol 37: 920-923, 2015.
9 Bhat A, Vijaya C and Rao SB: Pseudoangiomatous variant of spindle cell lipoma: Report of a rare case. Indian J Pathol Microbiol 59: 376-378, 2016.

10 Marks E, Amin SM, Xia L, Guitart J and Laskin W: Pseudoangiomatous spindle cell lipoma: a distinct subtype of spindle cell lipoma. Pathol Int 2016.

11 Mandahl N: Methods in solid tumour cytogenetics. In: Human Cytogenetics: Malignancy and Acquired Abnormalities. Rooney DE (ed.). New York: Oxford University Press, pp. 165-203, 2001.

12 McGowan-Jordan J, Simons A and Schmid M: ISCN 2016: An International System for Human Cytogenetic Nomenclature. Basel: Karger, 2016.

13 Austin CD, Tiessen JR, Gopalan A, Williams JM, Jr., Bangs CD, Cherry AM, Lehnert BA and Rouse RV: Spindle cell lipoma of the foot and the application of CD34 immunohistochemistry to atypical lipomatous tumors in unusual locations. Appl Immunohistochem Mol Morphol 8: 222-227, 2000.

14 Bartuma H, Nord KH, Macchia G, Isaksson M, Nilsson J, Domanski HA, Mandahl N and Mertens F: Gene expression and single nucleotide polymorphism array analyses of spindle cell lipomas and conventional lipomas with 13q14 deletion. Genes Chromosomes Cancer 50: 619-632, 2011.

15 Dahlén A, Debiec-Rychter M, Pedeutour F, Domanski HA, Höglund M, Bauer HC, Rydholm A, Sciot R, Mandahl N and Mertens F: Clustering of deletions on chromosome 13 in benign and lowmalignant lipomatous tumors. Int J Cancer 103: 616-623, 2003.

16 Dal Cin P, Sciot R, Polito P, Stas M, de Wever I, Cornelis A and Van den Berghe H: Lesions of $13 \mathrm{q}$ may occur independently of deletion of $16 \mathrm{q}$ in spindle cell/pleomorphic lipomas. Histopathology 31: 222-225, 1997.

17 Mandahl N, Mertens F, Willén H, Rydholm A, Brosjö O and Mitelman F: A new cytogenetic subgroup in lipomas: loss of chromosome 16 material in spindle cell and pleomorphic lipomas. J Cancer Res Clin Oncol 120: 707-711, 1994.

18 Rusconi D, Valtorta E, Rodeschini O, Giardino D, Lorenzo I, Predieri B, Losa M, Larizza L and Finelli P: Combined characterization of a pituitary adenoma and a subcutaneous lipoma in a MEN1 patient with a whole gene deletion. Cancer Genet 204: 309-315, 2011.

19 Walther C, Domanski HA, von Steyern FV, Mandahl N and Mertens F: Chromosome banding analysis of cells from fine-needle aspiration biopsy samples from soft tissue and bone tumors: Is it clinically meaningful? Cancer Genet 204: 203-206, 2011.

20 Maggiani F, Debiec-Rychter M, Vanbockrijck M and Sciot R: Cellular angiofibroma: another mesenchymal tumour with $13 \mathrm{q} 14$ involvement, suggesting a link with spindle cell lipoma and (extra)mammary myofibroblastoma. Histopathology 51: 410-412, 2007.

21 Hameed M, Clarke K, Amer HZ, Mahmet K and Aisner S: Cellular angiofibroma is genetically similar to spindle cell lipoma: a case report. Cancer Genet Cytogenet 177: 131-134, 2007.

22 Chen BJ, Marino-Enriquez A, Fletcher CD and Hornick JL: Loss of retinoblastoma protein expression in spindle cell/pleomorphic lipomas and cytogenetically related tumors: an immunohistochemical study with diagnostic implications. Am J Surg Pathol 36: 1119-1128, 2012. 